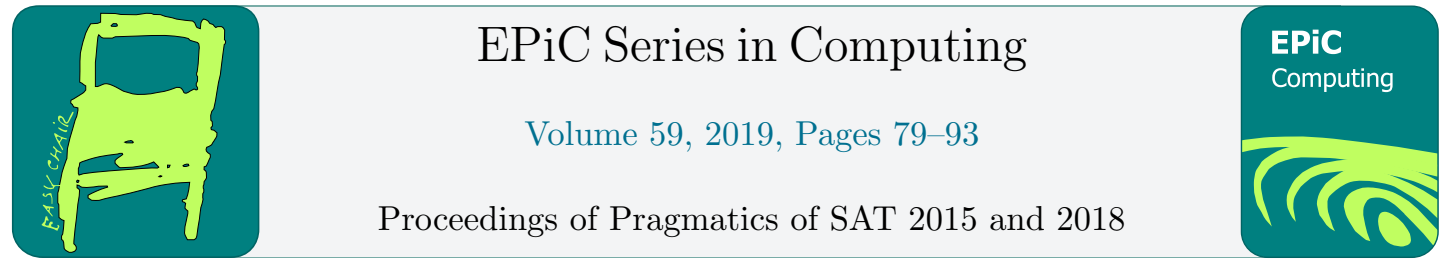

\title{
CryptoMiniSat Switches-Optimization for Solving Cryptographic Instances
}

\author{
A.-M. Leventi-Peetz ${ }^{1}$, O. Zendel ${ }^{1}$, W. Lennartz ${ }^{2}$, and K. Weber ${ }^{2}$ \\ ${ }^{1}$ Federal Office for Information Security, \\ Godesberger Allee 185-189, DE-53175 Bonn, Germany \\ leventi-peetz@bsi.bund.de \\ 2 inducto $\mathrm{GmbH}$, \\ Karl-Wastl-Straße 13, DE-84405 Dorfen, Germany
}

\begin{abstract}
Performing hundreds of test runs and a source-code analysis, we empirically identified improved parameter configurations for the CryptoMiniSat (CMS) 5 for solving cryptographic CNF instances originating from algebraic known-plaintext attacks on 3 rounds encryption of the Small AES-64 model cipher $\operatorname{SR}(3,4,4,4)$. We finally became able to reconstruct 64-bit long keys in under an hour real time which, to our knowledge, has never been achieved so far. Especially, not without any assumptions or previous knowledge of key-bits (for instance in the form of side-channels, as in [11]). A statistical analysis of the non-deterministic solver runtimes was carried out and command line parameter combinations were defined to yield best runtimes which ranged from under an hour to a few hours in median at the beginning. We proceeded using an Automatic Algorithm Configuration (AAC) tool to systematically extend the search for even better solver configurations with success to deliver even shorter solving times. In this work we elaborate on the systematics we followed to reach our results in a traceable and reproducible way. The ultimate focus of our investigations is to find out if CMS, when appropriately tuned, is indeed capable to attack even bigger and harder problems than the here solved ones. For the domain of cryptographic research, the duration of the solving time plays an inferior role as compared to the practical feasibility of finding a solution to the problem. The perspective scalability of the here presented results is the object of further investigations.
\end{abstract}

\section{Introduction}

CryptoMiniSat ${ }^{1}$ offers a wide range of parameter settings to choose when calling the solver and these parameters seem to sensitively influence the search for a solution in case of cryptographic CNF (conjunctive normal form) instances. We selected parameter combinations that especially affect the solver runtime-behavior in the case of CNF instances, generated from algebraic equations systems that represent known-plaintext attacks (KPA) on the Small AES-64 model cipher. The KPA is an attack model of cryptanalysis where the attacker has access to both the plaintext and its encrypted version (ciphertext). Knowing the cryptographic algorithm, the goal of

\footnotetext{
${ }^{1}$ Developed by Mate Soos as an open source community project [13, 12].
} 
the attack is to reconstruct the secret key from the text information. AES is an iterated block cipher which, as the encryption mechanism, repeats a set of state transformations for a number of rounds. The input (plaintext) and the output of each and every transformation is called state and consists of a fixed number of bits. AES makes use of both non-linear and linear transformations, one being the round-key addition. The round keys are generated from the secret key. To perform the state transformations, the state or block is divided into words which are arranged in a rectangular array, structured by rows and columns. In AES words are eight bits long. The nonlinear transformation operates on each word independently. For the key recovery one first describes the encryption algorithm in the form of a Boolean MQ (multi quadratic) polynomial equation system of bit variables, as introduced by Courtois and Pieprzyk [3]. Inserting the known bits of the plaintext and cyphertext pairs one gets a non-linear system of equations, the solution of which delivers the secret key. Each additional text pair adds another set of equations to the system but with the same key bit variables. The equations of the round key expansion enter only once. This system can be tackled with algorithms based on Gröbner bases and SAT solvers or alternatively only with a SAT solver, which is the approach followed here. Routinely, so named small scale variants of the AES polynomial system [2] are employed for tests in the cryptographic community. Relevant to the usual variants are the following numbers:

- $n$ is the number of (encryption) rounds,

$-r$ is the number of rows in the rectangular arrangement of the state,

$-c$ is the number of columns in the rectangular arrangement of the state,

$-e$ is the size (in bits) of a word, normally 4 or 8 .

AES would be considered as broken when the model for $(n, r, c, e)=(10,4,4,8)$ has been solved and the corresponding 128-bit long key has been recovered. However, already successes to recover 8-bit and 16-bit long keys for very small AES model ciphers are reported in the literature, mainly in association with benchmarking of SAT solvers in comparison to one another $[1,8]$. Here the solution of the model system $\operatorname{SR}(3,4,4,4)$ is discussed using CMS.

The computations have been performed on both four socket AMD Opteron 6378 and two socket EPYC 7551 systems with 256 GB RAM using 31 threads per job (64 respectively 128 would have been possible) and up to four jobs parallelly. Details concerning the derivation of the $\mathrm{MQ}$ algebraic equations system of the attack and its transformation to CNF will not be further discussed here because the focus of this work lies on the configuration of the solver. The interested reader can find some of these information in [9]. CryptoMiniSat solves all cases of 2 rounds encryption, that is the $\mathrm{SR}(2,4,4,4)$ case, for the Small AES-64 model within seconds. Key recovery from 3 rounds encryption can get successfully accomplished with the solver running in default parameter setting, however mostly within hours. The solution finding is subjected to distinct statistical variations, due to the indeterministic behavior of the solver in multi-thread operation mode. When an upper runtime-limit has been set, it is a matter of chance whether the solver will find the solution or not. Running the solver determininistically in single-thread modus is out of the question because it takes infeasible long. The parallely and asynchronously running solver threads complement each other by exchanging information and are indispensable for finding the solution in tolerable time. A systematical statistical investigation of the solver's behavior for a multitude of cases helped us find solver parameter combinations which enable key recovery for 3 rounds AES-64 encryption, or solving the $\operatorname{SR}(3,4,4,4)$ case, within predictable time-intervals. We extended our efforts beyond the empirical parameter optimization by employing an automatic algorithm configuration tool which we adapted for the problem and applied it to find even better parameter settings. 
This paper is organized in 5 parts as follows: In the first part we give an overview of the size, format and the density of the CNF instances which we have worked with. In the second part we present and discuss runtime statistics of the solver in its default parameter settings. In the third part the empirical parameter optimization investigations and their results are presented and discussed. Due to the indeterministic behavior of the solver in multi-thread operation mode, certain changes in the source code have been undertaken, which were seen as necessary in order to increase the significance of the influence of changes in parameter settings to the benefit of the generation of distinct results. These source-code changes will be substantiated and the out of them resultant improvements of the solver runtimes will be graphically demonstrated. In the fourth part of this paper, the innovative results of an Automatic Algorithm Configuration for the parameters of CryptoMiniSat which produced even better parameter configurations will be presented and discussed. We conclude with a summary and description of further planned investigations to optimize CMS.

\section{Classification of CNF-Instances}

The plaintexts were made of english words and spaces. We have varied the number of the KPA text pairs, used for the instances generation, in order to investigate also the influence of this number on the solution runtime for instances otherwise created with the same key. The number of text pairs measures the redundancy of information given to the solver, as all texts are encoded with the same algorithm (the same logic) and the same key. The bigger the number of text pairs, the bigger the number of variables and constraints in the resulting instance to solve, so one shouldn't expect to be able to always get an advantage by arbitrarily increasing the number of text pairs. However, for each key case there seems to exists an optimal number of text pairs minimizing the solution time and that has to be discovered. The number of text pairs varied here between 16 and 32 pairs. It should be noted that these cryptographic CNF instances only possess one truth assignment by construction. An overview of the parameters of the tested CNF instances is listed in table 1. We varied also the quality of the encoding key. Experiments were performed with several different keys, belonging to three different classes. As representative of each class, three keys are listed below, with which the results presented here have been produced.

k4 '0101010101010101'; pathologic or insecure

k3 '0123456789abcdef'; structured

k6 'b25286f7d3e7b3e1'; secure, random

All instances contain clauses of varying length and all instances are of the type sparse and without inclusion of explicit XOR-Clauses.

\section{Runtime Statistics for CMS in Default-Setting}

The CMS threads work asynchronously and the order in which they exchange information is unpredictable depending on external influences like the operating system and administrative tasks running on the computer. Similarly indeterministic and irreproducible is each and every solver run and solution process. This circumstance leads to the result that repeated solver attempts to solve one and the same instance under identical parameter configuration can deliver very different runtimes, which renders the nature of statements about average runtime 
Table 1: Some characteristic numbers of the utilized CNF instances. Instance tokens comprise $\langle$ no. of rounds $\rangle-\langle k e y$ token $\rangle-\langle$ no. of text pairs $\rangle$.

\begin{tabular}{|c|c|c|c|c|c|}
\hline Instance $^{a}$ & Rounds & Text Pairs & Variables $L$ & Clauses $N$ & Density $N / L$ \\
\hline $3-\mathrm{k} 4-12$ & 3 & 12 & 4096 & 1228120 & 299.8 \\
\hline $3-\mathrm{k} 4-16$ & 3 & 16 & 5376 & 1626619 & 302.6 \\
\hline $3-\mathrm{k} 4-30$ & 3 & 30 & 9856 & 3021481 & 306.6 \\
\hline $3-\mathrm{k} 3-12$ & 3 & 12 & 4096 & 1227940 & 299.8 \\
\hline $3-\mathrm{k} 3-14$ & 3 & 14 & 4736 & 1427202 & 301.4 \\
\hline $3-\mathrm{k} 3-18$ & 3 & 18 & 6016 & 1825395 & 303.4 \\
\hline $3-\mathrm{k} 3-20$ & 3 & 20 & 6656 & 2024600 & 304.2 \\
\hline $3-\mathrm{k} 3-22$ & 3 & 22 & 7296 & 2224391 & 304.9 \\
\hline $3-\mathrm{k} 3-30$ & 3 & 30 & 9856 & 3021481 & 306.6 \\
\hline $3-\mathrm{k} 6 \mathrm{~s}-20$ & 3 & 20 & 6656 & 2025228 & 304.3 \\
\hline $3-\mathrm{k} 6 \mathrm{~s}-22$ & 3 & 22 & 7296 & 2224391 & 304.9 \\
\hline $3-\mathrm{k} 6 \mathrm{~s}-24$ & 3 & 24 & 7936 & 2424008 & 305.4 \\
\hline $3-\mathrm{k} 6 \mathrm{~s}-30$ & 3 & 30 & 9856 & 3021481 & 306.6 \\
\hline $4-\mathrm{k} 6 \mathrm{~s}-30$ & 4 & 30 & 13760 & 4447760 & 323.2 \\
\hline
\end{tabular}

${ }^{a}$ k3: 0123456789abcdef; k4: 0101010101010101; k6: b25286f7d3e7b3e1

measurements to a statistical one. Boxplots ${ }^{2}$ have been chosen as proper statistical analysis method for the runtime measurements.

In Figure 1 the runtime analysis of the solver for the as insecure classified key for 12, 16, and 30 text pairs respectively is depicted. The median of the runtime varies with the number of text pairs and the faster key recovery is achieved with the instance created out of 16 text pairs. Also the mean values of the measured data reflect the same result though each assuming a higher value than its respective median. In Figure 2 there is depicted the runtime analysis of the solver for the structured key case and for 12, 14, 18, 20, 22, and 30 text pairs, respectively. Also in this case does the median of the solution time distinctly vary dependent on the number of text pairs, the optimal number appearing to be this time in the case of 22 pairs. Again mean values and medians stay consistent to this result with the mean values climbing a bit higher than the medians. Obviously the use of the structured key makes the solution of the problem considerably more expensive shifting the solution time one order of magnitude towards higher values. In plot 3 the solver runtime analysis regarding the solution of instances created with the random or secure key is demonstrated. The solution runtime is of the same order of magnitude as in the case of the structured, or simple key, the optimal number of text pairs appearing to be 24 this time. The CMS runtime measurements produce a spectrum of random data representing solver runtimes containing some few extremely long runtimes. However, in each case the majority of the resulting values lie within a well defined limited region.

Comparing the solution times in the plots, one observes that the number of text pairs is important, because a convenient choice of this number can occasionally strongly diminish the solver runtime for instances otherwise created with the same key. Comparing solution runtimes for three different keys using instances created with the same number of text pairs, one can attest that a simple key costs a shorter solution runtime as compared to the runtime needed to solve the instance generated with a secure key, see Figure 4. In table 2 the runtime statistics for the calculations with the CMS in default setting are portrayed.

\footnotetext{
${ }^{2}$ See for example WiKIPEDIA Box plot https://en.wikipedia.org/wiki/Box_plot.
} 


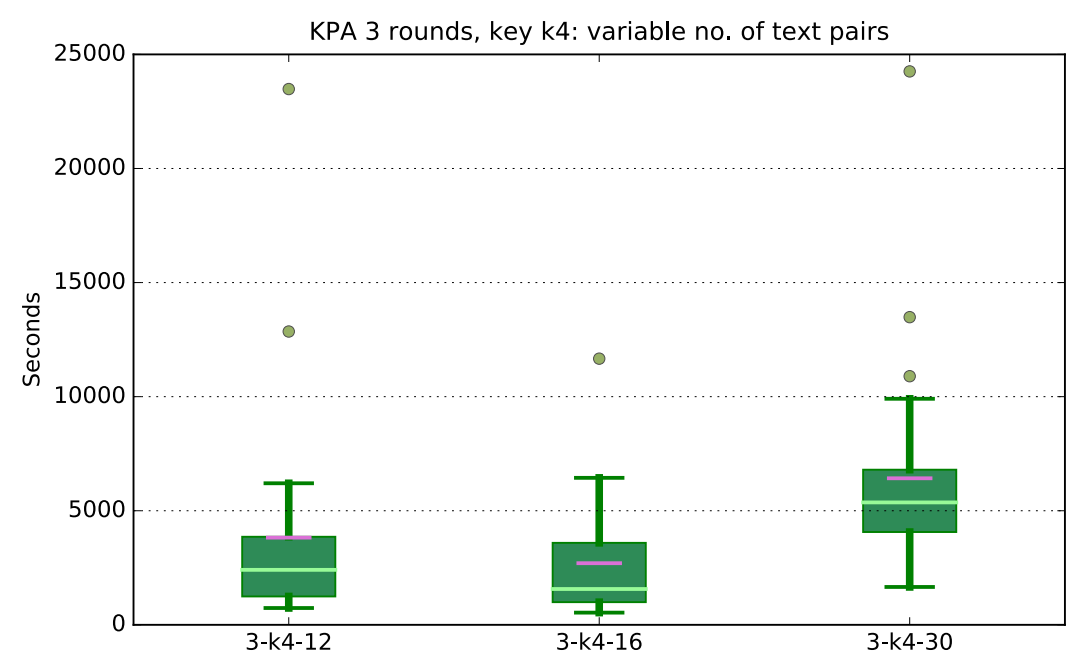

Figure 1: 3 rounds, key k4: varying number of text pairs.

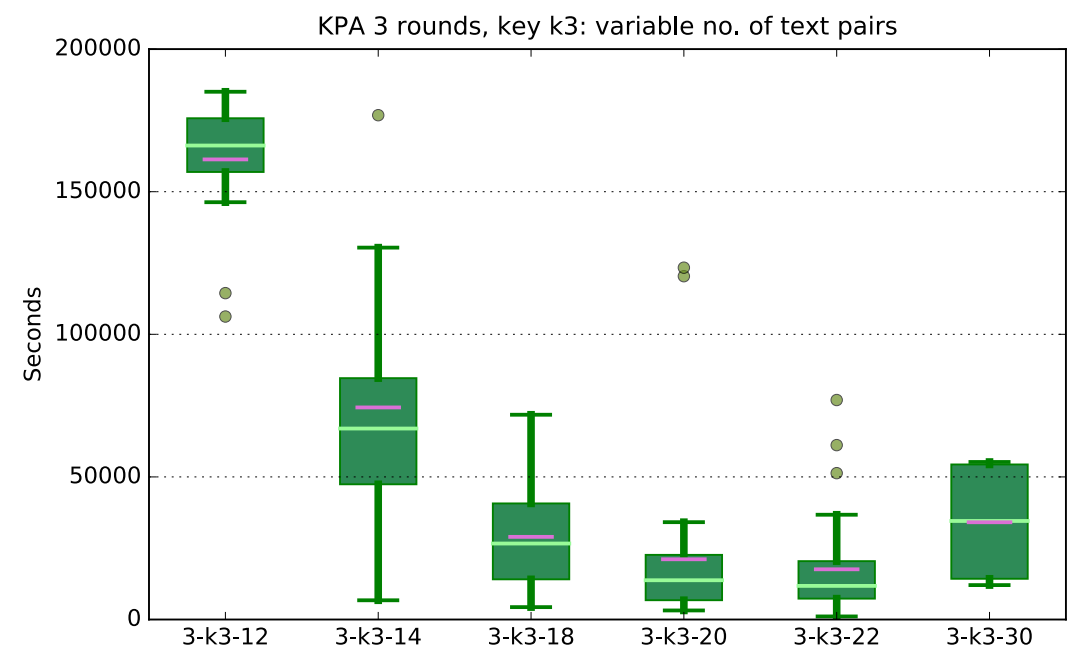

Figure 2: 3 rounds, key k3: varying number of text pairs.

\section{Empirical Parameter Optimization}

A dynamic code-analysis of the solver preceded the practical parameter optimization phase, so as to investigate how the solver-runtime consumption is distributed between the various solver sub-processes and functions dependent on the external parameter settings and the instance to solve. Again the dynamic analysis results are of statistical character, as the various solver modules and functions are regularly called many times during the solver runtime. A previous static code analysis had provided associations between external parameters and according parts of the code. The question was, if dependent on the problem at hand, one could possibly discover 


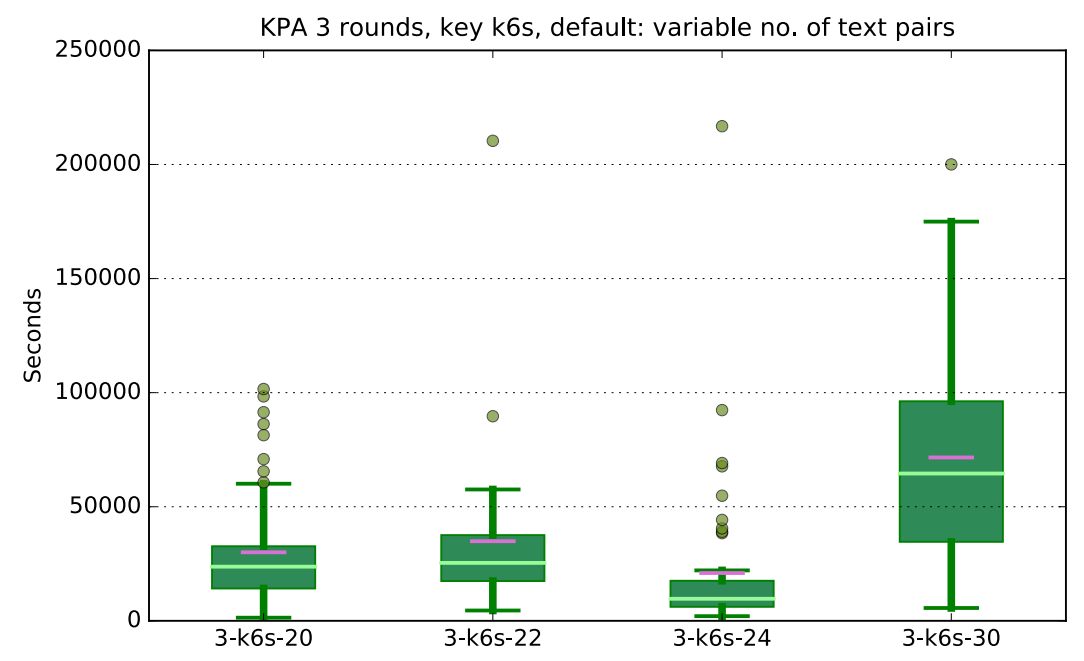

Figure 3: 3 rounds, key k6s: varying number of text pairs.

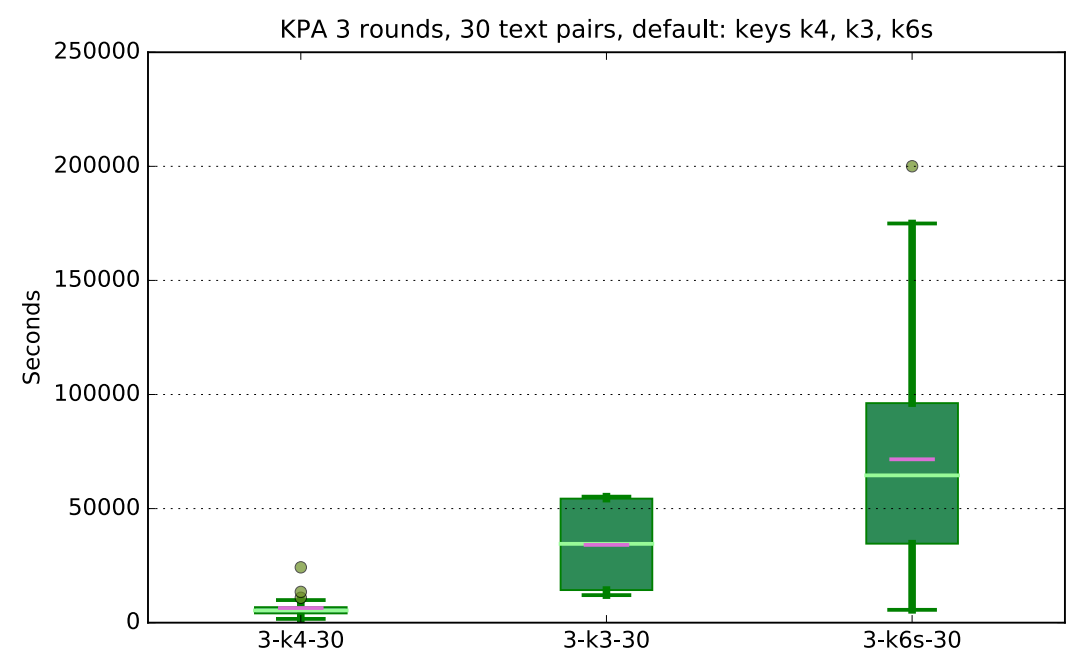

Figure 4: 3 rounds, constant number of text pairs, varying keys.

some optimal strategy of how to vary on parameters influencing favorably the execution of the most time-consuming code parts, so as to effectively shorten the solution runtime. For profiling the CMS program we used the GNU/Linux perf tool ${ }^{3}$. Flame Graphs generated with the open source tool of the same name developed by Brendan Gregg [5] are utilized for the visualization of the profiling results. The code performance profiling has been carried out in both default solver setting and with certain parameter settings other than default. This delivered converging and unique results, as regards those parts of the code causing the greatest CPU-load in case

\footnotetext{
${ }^{3}$ See for example WiKIPEDIA perf (Linux) https://en.wikipedia.org/wiki/Perf_(Linux).
} 
Table 2: Runtime statistics with CMS in default setting. The instance token comprises $\langle$ number of rounds $\rangle-\langle k e y$ token $\rangle-\langle$ number of text pairs $\rangle$.

\begin{tabular}{|c|c|c|c|c|c|c|}
\hline Instance $^{a}$ & count & median & \multicolumn{2}{|c|}{ quartile } & mean & $\sigma[\%]$ \\
\hline $3-\mathrm{k} 4-12$ & 25 & 2409.6 & 1243.8 & 3859.6 & 3821.9 & 126 \\
\hline $3-\mathrm{k} 4-16$ & 25 & 1569.8 & 993.6 & 3592.8 & 2700.8 & 98 \\
\hline $3-\mathrm{k} 4-30$ & 26 & 5281.6 & 4008.6 & 6221.2 & 5736.2 & 47 \\
\hline 3-k3-12 & 17 & 167460.0 & 157160.3 & 175973.7 & 167778.0 & 21 \\
\hline 3-k3-14 & 15 & 66963.4 & 47415.0 & 84625.9 & 74358.1 & 58 \\
\hline $3-\mathrm{k} 3-18$ & 11 & 26645.1 & 14093.4 & 40702.4 & 28979.0 & 71 \\
\hline 3-k3-20 & 31 & 14073.1 & 6818.8 & 23159.6 & 27382.6 & 163 \\
\hline $3-\mathrm{k} 3-20^{b}$ & 8 & 56141.5 & 16057.7 & 105700.4 & 70640.1 & 98 \\
\hline 3-k3-22 & 36 & 11788.1 & 7328.1 & 20468.9 & 17610.4 & 95 \\
\hline $3-\mathrm{k} 3-30$ & 4 & 34551.3 & 14286.1 & 54366.9 & 34101.8 & 70 \\
\hline $3-\mathrm{k} 6 \mathrm{~s}-20$ & 50 & 23925.9 & 14190.5 & 34829.1 & 33958.6 & 112 \\
\hline $3-\mathrm{k} 6 \mathrm{~s}-22$ & 33 & 26602.2 & 17846.1 & 45668.5 & 54138.4 & 157 \\
\hline $3-\mathrm{k} 6 \mathrm{~s}-24$ & 51 & 9462.4 & 6100.0 & 17050.3 & 17113.6 & 111 \\
\hline $3-\mathrm{k} 6 \mathrm{~s}-30$ & 22 & 64556.1 & 34625.1 & 96221.0 & 71600.6 & 76 \\
\hline
\end{tabular}

\footnotetext{
${ }^{a}$ k3: 0123456789abcdef; k4: 0101010101010101; k6: b25286f7d3e7b3e1

${ }^{b}$ Solution calculated with 21 threads.
}

of all instances implementing the attack on the 3 rounds encryption of the AES-64 model cipher. These results have been verified against different encryption keys and different numbers of text pairs employed for the generation of the problem instances and they appear to be stable. $82 \%$ to $97 \%$ of the runtime the solver invests into its search routine (especially, the method propagate_any_order_fast() of the propagation procedure) and this is independent of whether an instance terminates during the observed runtime or not.

Since the solution of the considered CNF instances typically takes a few thousand seconds, the profiling was performed only during a part of the program run. To insure a stable result, we chose the measuring interval big enough, as well as the sampling frequency $f$ for perf high enough. One has to keep in mind that the performance profiling for CMS delivers a statistical statement. The reasons are: 1) the program runs of CMS are indeterministic; 2) in multithread modus of CMS, the profiling averages over all threads where the threads are computing independently of each other at different parts of the code; 3) the interval of the profiling measurement starts at different times of the running program.

Exemplarily, a first profiling visualization is depicted in Figure 5, where the relative runtimeshares which the most used code paths of CMS consume, are depicted for the case of the medium hard to solve CNF instance 3-k3-22. Apart from the verbosity level and the multi-thread mode, CMS was here applied in default configuration.

Of interest is the variation of parameters influencing the whole of the search process or such that influence the function propagate_any_order_fast () in a direct way. In table 3 there are listed parameters influencing the solver's restart process, the choice of variables and the setting of the so called glue values. The parameter --updateglueonprop is the only one directly affecting the function propagate_any_order_fast(). The last two parameter combinations, sw3 and sw4, indicate calculations with a modified version of CMS. In the multi-thread mode, CMS configures most threads slightly different than the command-line settings in order to deliver good performance with the variety of CNF instances to be solved in SAT solver competitions. 


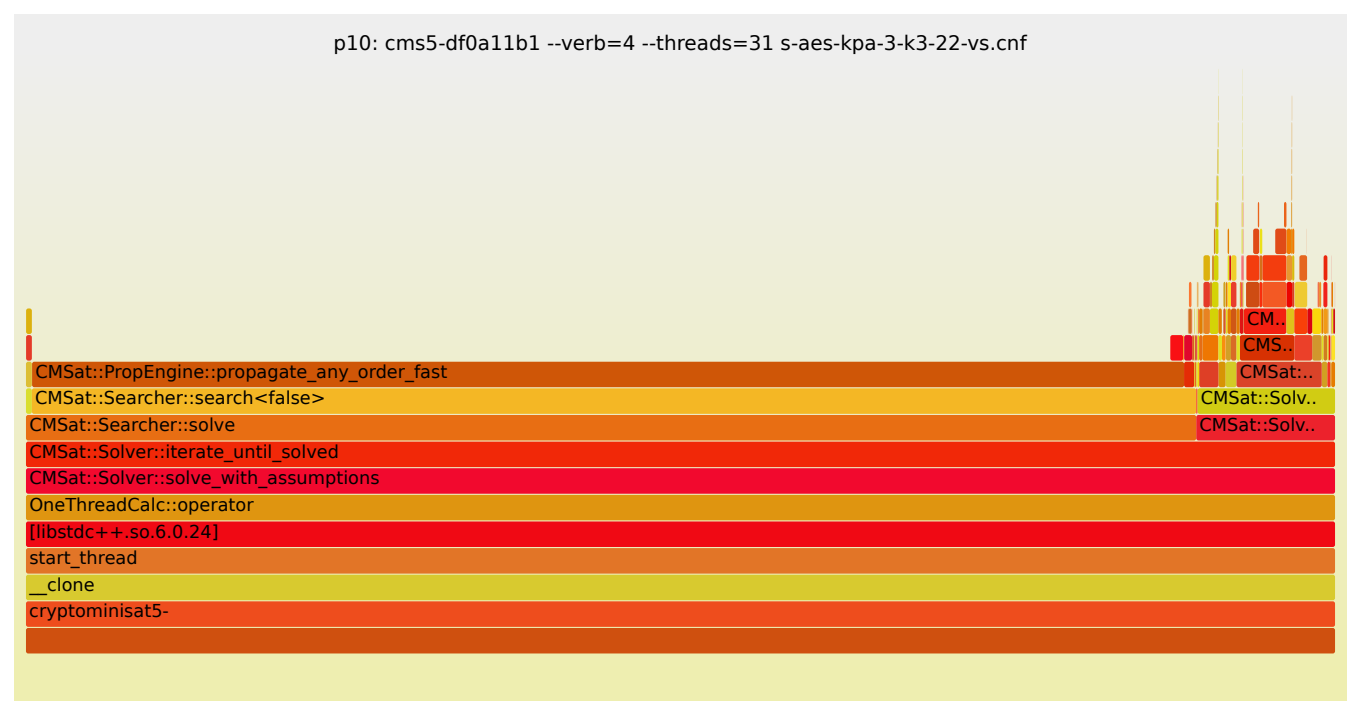

Figure 5: FlameGraph 3 rounds: 3-k3-22 with 31 threads, default parameter configuration, performance measuring with $f=250 \mathrm{~Hz}$ for $300 \mathrm{~s}$.

Table 3: Explored parameter combinations and their abbreviations. Also set for all CMS runs: --verb $=4$--threads $=31$--comps $=0$. sw3 und sw4 imply the usage of the here modified CMS.

\begin{tabular}{|c|c|}
\hline Abbreviation & Parameter Combinations \\
\hline sw1 & --restart=geom --maple $=1--$ bva $=0 \quad--$ sync $=30000$ \\
\hline sw2 & --gluehist $=30--$ maple $=1--$ maxnummatrixes $=8 \quad-$-bva $=0$ \\
\hline sw6 & -- restart $=$ glue - -gluecut $0=4--$ updateglueonprop $=1$ \\
\hline sw7 & --gluecut $0=5$--gluecut $1=7$--updateglueonprop $=1$ \\
\hline sw3 & --restart=geom --maple $=1--$ cachesize $=4096--$ cachecutof $f=3000$ \\
\hline sw4 & --restart=glue --gluecut0 $=4$--updateglueonprop $=1$ \\
\hline
\end{tabular}

To enhance the influence of the here considered parameter combinations on the solution of the examined cryptographic CNF instances, we changed the source code so that the solver uses the same command-line settings for all threads. Only the pseudo random number generator (PRNG) of each thread is seeded differently. This change is effective, because it limits the solver's strategy to one exclusively examined configuration whereat the threads get diversified due to the usage of the PRNG and the asynchronous information exchange.

In the FlameGraph-visualization of Figure 6 there are depicted the relative runtime-shares which the most used code paths of CMS consume during the calculations for the harder to solve CNF instance 3-k6s-30. In this case the parameter combination sw4 was employed. Here the solver spends a bit less time in the search as compared to the the case shown in figure 5 .

Because instances created with the insecure key $\mathrm{k} 4$ could be solved relatively fast with the CMS in default setting, only instances created with the other two keys have been employed for the parameter optimization tests. In Figure 7 the solver runtime analysis for solving instances created with the structured key $\mathrm{k} 3$ is demonstrated for different parameter combinations. In the next Figure 8 the same instance as in Figure 7 is tested with the parameter combinations: default, sw3 and sw4. Evidently the choice of parameter combinations has a considerable 


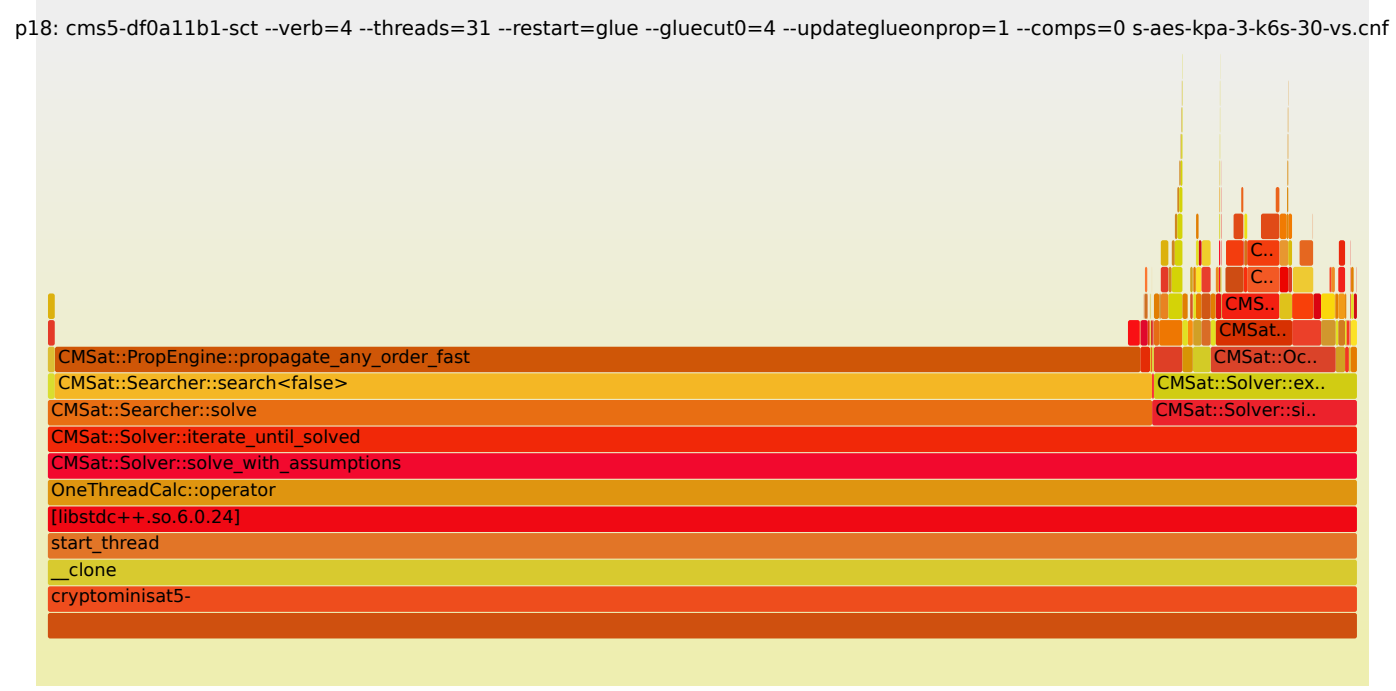

Figure 6: FlameGraph 3 rounds: 3-k6s-30 with 31 threads, parameter configuration sw4, performance measuring with $f=250 \mathrm{~Hz}$ for $300 \mathrm{~s}$.

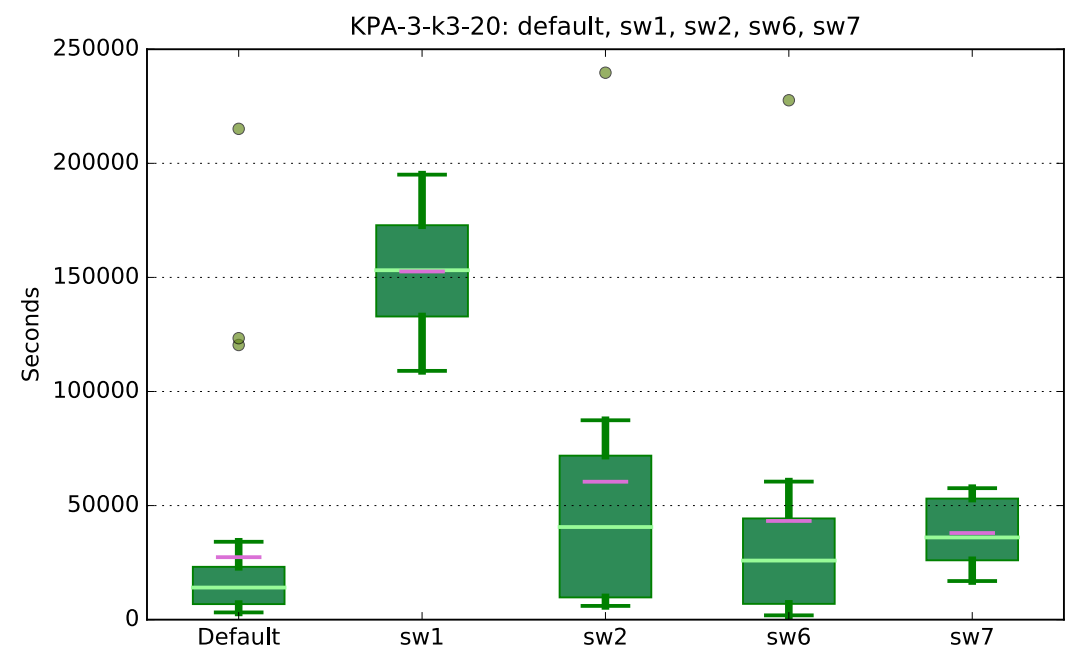

Figure 7: 3 rounds, key k3: 20 pair texts, parameter combinations: default, sw1, sw2, sw6, sw7.

influence on the solution runtime of the solver. An improvement in the solution time is registered with the sw4 parameter combination as compared to the default setting. Changing the number of text pairs we test the stability of the sw4 runtime advantage, as a function of the number of text pairs. We observe that this advantage can get attenuated or amplified, dependent on the chosen number of text pairs, see Figure 9. This suggests that the number of text pairs should also be observed as a problem optimization parameter. There follows a series of runtime tests with sw4, solving instances created with the secure key k6. The results are exhibited in Figure 10. In Figure 10 we see that the instance created with 30 text pairs allows the faster 


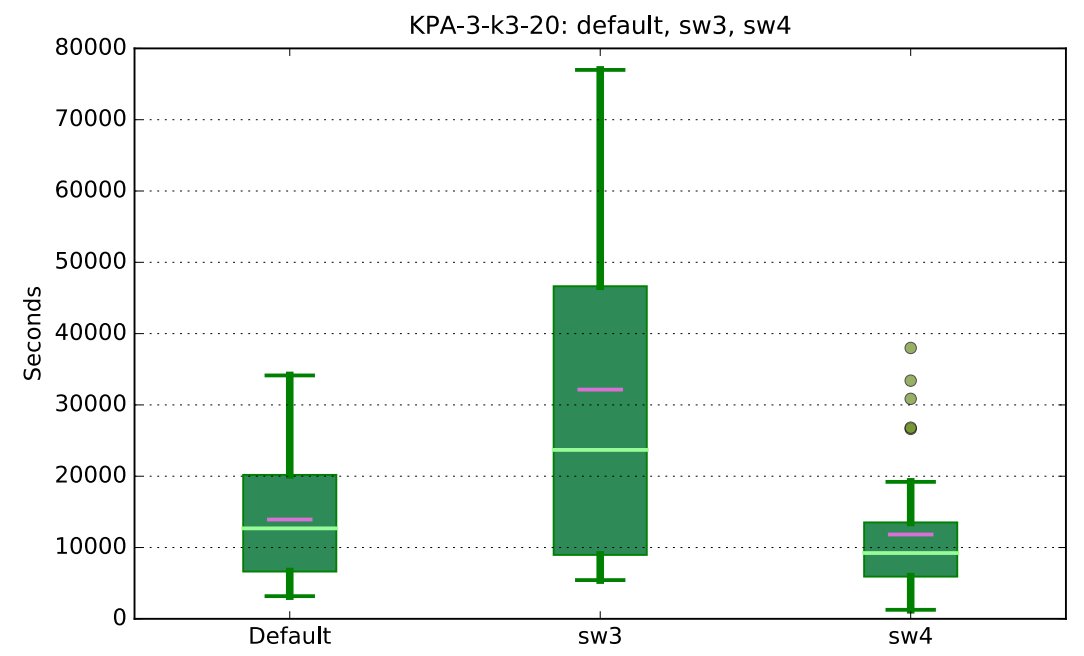

Figure 8: 3 rounds, key k3: 20 pair texts, parameter combinations: default, sw3 and sw4.

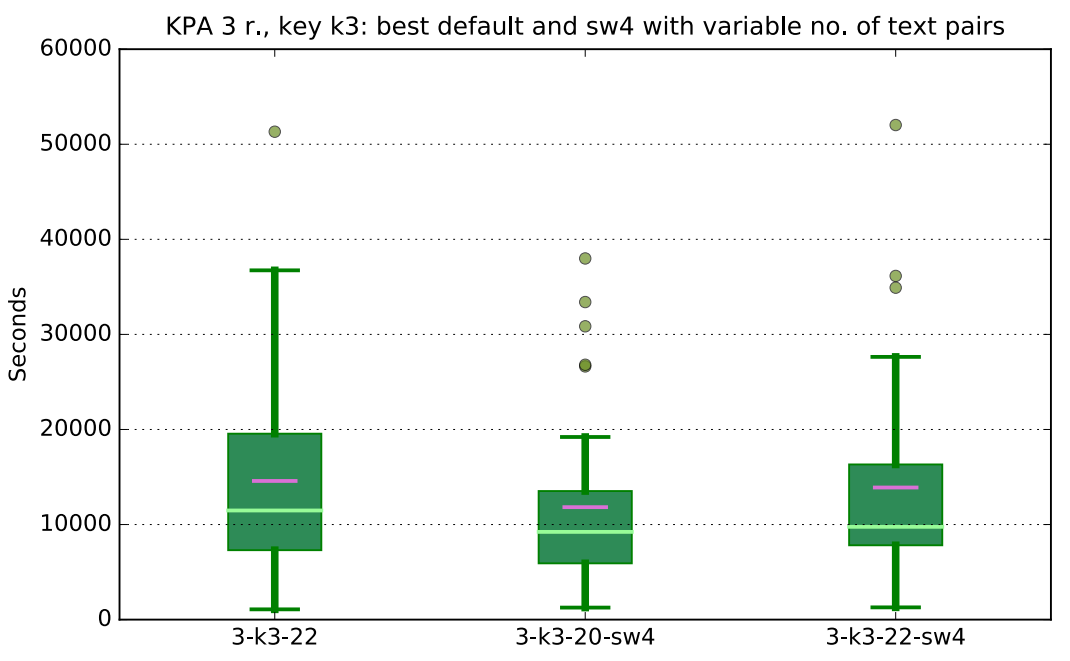

Figure 9: 3 rounds, key k3: best default, and sw4 with variable number of text pairs.

reconstruction of the key $\mathrm{k} 6$ when the parameter combination sw4 is applied. Notably, even the upper quartile of the optimized best solver runtime, for the instance with the 30 text pairs, lies underneath the lower quartiles of the runtimes of all other instances, thus establishing the unambiguity of this result. The following Boxplot in Figure 11 depicts the comparison between the best results for all three different keys. It seems that for every key and independent of its security quality, there exists a combination of a number of text pairs and a CMS configuration to find the solution with a statistical median lying well below the 10000 seconds runtime limit for the solver. This is a significant result because it indicates that a secure key might not necessarily offer better protection against solving the here discussed instances. In table 4 the 


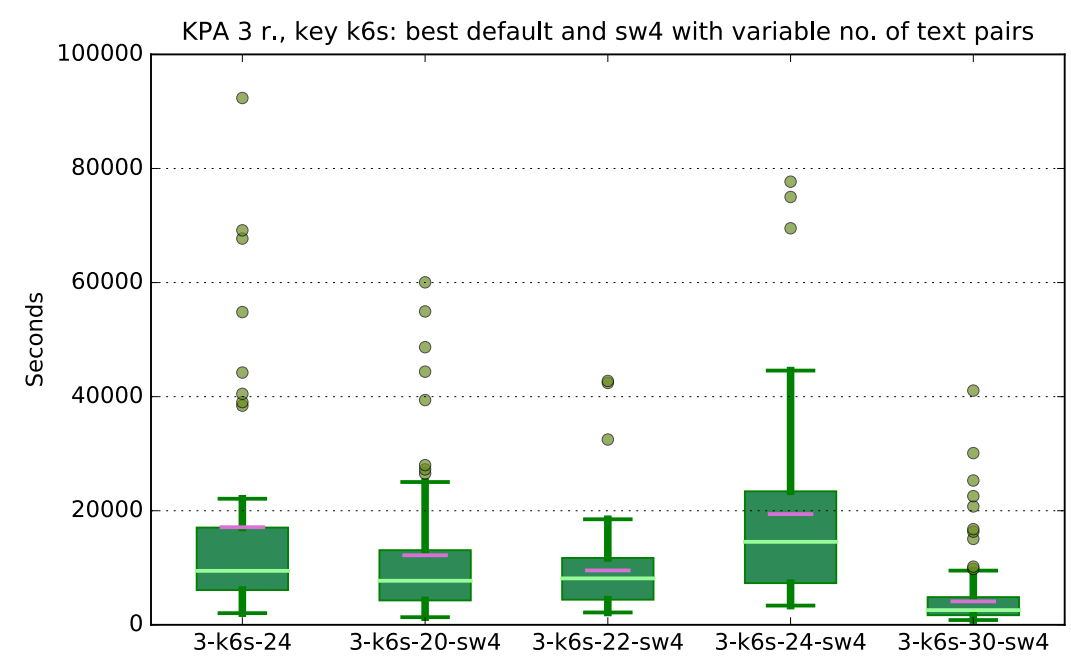

Figure 10: 3 rounds, key k6: best default, and sw4 with variable number of text pairs.

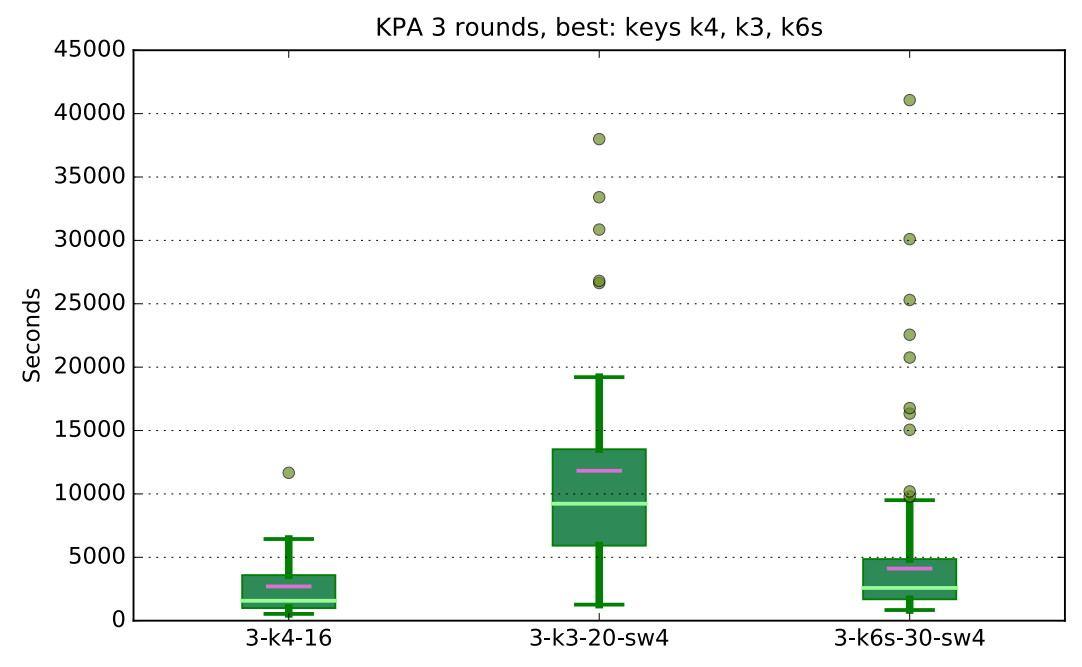

Figure 11: 3 rounds best for keys: k4, k3, k6s.

solver runtime statistics for the practically optimized parameter settings are presented.

\section{Automatic Algorithm Configuration (AAC)}

The adaption of SAT solver configurations to a specific type of instances or instance classes is a common practice employed by many developers of such programs participating in the international SAT solver competition. ${ }^{4}$ Meanwhile, also computer tools for the automatic algorithm

\footnotetext{
${ }^{4}$ International SAT Solver Competitions http://www . satcompetition.org.
} 
Table 4: Runtime statistics with various parameter combinations for CMS. Instance tokens comprise $\langle$ no. of rounds $\rangle-\langle k e y$ token $\rangle-\langle$ no. of text pairs $\rangle$.

\begin{tabular}{c|r|r|r|r|r|r}
\hline Instance $^{a}$ & count & median & \multicolumn{2}{|c|}{ quartile } & mean & $\sigma[\%]$ \\
\hline 3-k3-20-sw1 & 4 & 153149.7 & 132870.1 & 172872.3 & 152592.8 & 24 \\
3-k3-20-sw2 & 9 & 40596.2 & 9753.6 & 71882.5 & 60456.5 & 121 \\
3-k3-20-sw3 & 12 & 23689.6 & 8963.1 & 46646.4 & 32149.3 & 87 \\
3-k3-20-sw4 & 35 & 9227.2 & 5919.9 & 13522.9 & 11828.3 & 79 \\
3-k3-20-sw6 & 11 & 25895.9 & 6922.7 & 44363.8 & 43264.8 & 148 \\
3-k3-20-sw7 & 8 & 36036.9 & 26012.9 & 53067.9 & 37975.0 & 42 \\
3-k3-22-sw4 & 35 & 9761.5 & 7819.0 & 16320.9 & 13895.4 & 79 \\
3-k6s-20-sw4 & 60 & 7575.2 & 4225.7 & 12310.2 & 10686.0 & 95 \\
3-k6s-22-sw4 & 74 & 8142.4 & 4410.4 & 11720.8 & 9535.7 & 80 \\
3-k6s-24-sw4 & 59 & 14547.9 & 7312.2 & 23404.8 & 19408.2 & 88 \\
3-k6s-30-sw4 & 197 & 2581.0 & 1687.8 & 4857.5 & 4110.9 & 116 \\
\hline
\end{tabular}

${ }^{a}$ k3: 0123456789abcdef; k6: b25286f7d3e7b3e1

configuration are available. Such programs even participate in the Configurable SAT Solver Challenge (CSSC), organized by F. Hutter et al. [7]. Led by the results of the CSSC 2016 we chose the tool SMACv3, developed by M. Lindauer, F. Hutter et al. [10,6] at the Universities of Freiburg and British Columbia, to further optimize the parameter settings of CMS for cryptographic CNF instances.

In order to apply SMACv3 with CMS, we set up the required Python environment and implemented a Target Algorithm Evaluator (TAE), a Python wrapper around CMS version 5.0.1, for the SMACv3 optimization API. We defined the legal ranges of the parameters to be optimized by SMACv3 by setting up a Parameter Configuration Space (PCS). From the PCS, SMACv3 chooses parameter combinations and calls the TAE with it. The resulting runtime of a CMS computation returned via the TAE is evaluated by SMACv3 for the parameter optimization and for further calls of the TAE. SMACv3 is able to deal with indeterministic runtimes by repeatedly calling CMS with the same parameter configuration and evaluating an estimator for the runtime. We performed the optimization by using SMACv3 in parallel mode.

All parameter optimization runs were performed with the modified version of the CMS code with alike configuration for all threads. Starting from our empirical configuration results, we confined the parameter optimization to few CMS parameters, a typical PCS file of ours looks like follows:

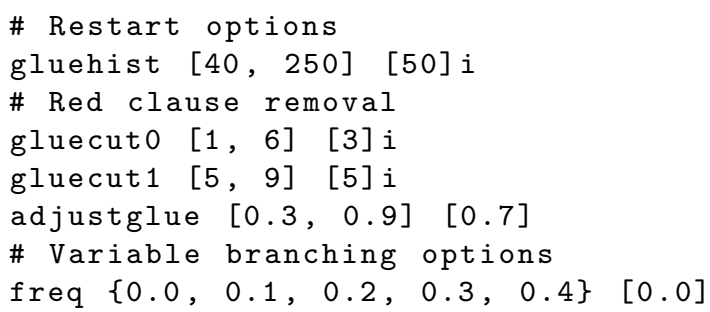

We performed optimizations for the CNF instances $3 \mathrm{k} 3-22$ and 3-k6s-30, independently. In Figures 12 and 13 we compare some intermediate states of the optimization (called incumbents by SMACv3) with the empirical best configuration sw4. From the results of several optimization runs (each computing nearly a week) we excerpted a configuration which reduces 


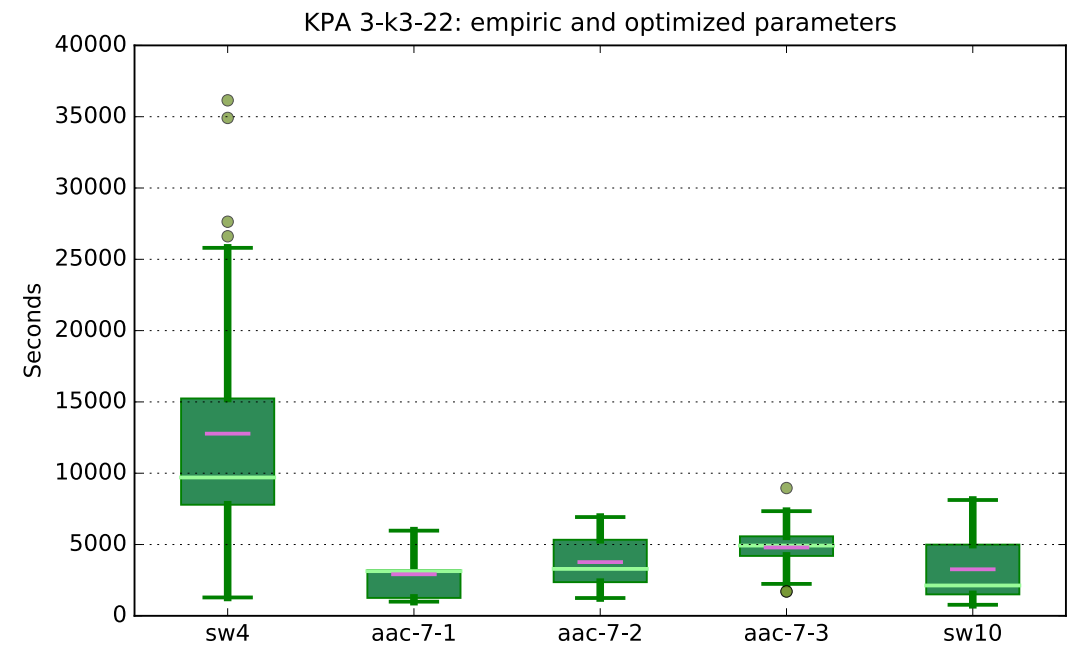

Figure 12: 3 rounds, key k3, 22 text pairs: sw4, 3 optimizer incumbents, and sw10.

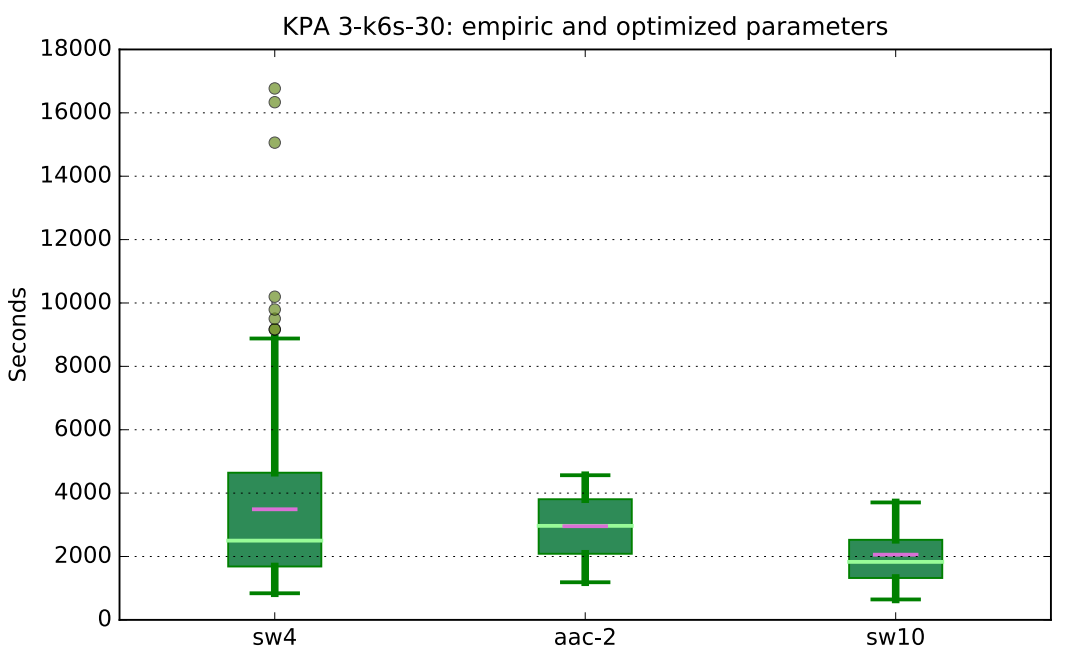

Figure 13: 3 rounds, key k6s, 30 text pairs: sw4, an optimizer incumbent, and sw10.

the median runtime for both instances. Compared to the empirical parameter setting sw4, the new best parameter combination sw10 sets additionally --gluecut1=7 --gluehist 45 . The Boxplots of the runtime analysis for this parameter setting are also shown in Figures 12 and 13. The numerical values of the Boxplot estimators are recapitulated in table 5 . The runtime of the CNF instance $3-\mathrm{k} 6 \mathrm{~s}-30$ could be improved by almost $30 \%$ and that of the instance $3-\mathrm{k} 3-22$ even by nearly $80 \%$ in median. 
Table 5: Runtime statistics of better parameter combinations for CMS. Instance tokens comprise $\langle$ no. of rounds $\rangle-\langle k e y$ token $\rangle-\langle$ no. of text pairs $\rangle$. Times are in seconds for the solving-thread.

\begin{tabular}{c|r|r|r|r|r|r}
\hline Instance $^{a}$ & count & median & \multicolumn{2}{|c|}{ quartile } & mean & $\sigma[\%]$ \\
\hline 3-k3-22-sw4 & 35 & 9761.5 & 7819.0 & 16320.9 & 13895.4 & 79 \\
3-k3-22-aac-7-1 & 5 & 3134.1 & 1256.4 & 3212.2 & 2913.7 & 68 \\
3-k3-22-aac-7-2 & 10 & 3291.5 & 2360.9 & 5333.9 & 3767.1 & 55 \\
3-k3-22-aac-7-3 & 25 & 4925.2 & 4201.4 & 5574.6 & 4792.0 & 35 \\
3-k3-22-sw10 & 15 & 2127.3 & 1503.9 & 4993.0 & 3262.4 & 72 \\
\hline 3-k6s-30-sw4 & 197 & 2581.0 & 1687.8 & 4857.5 & 4110.9 & 116 \\
3-k6s-30-aac-2 & 8 & 2966.6 & 2085.9 & 3807.3 & 2956.1 & 41 \\
3-k6s-30-sw10 & 15 & 1830.1 & 1321.5 & 2526.4 & 2057.4 & 46 \\
\hline
\end{tabular}

${ }^{a}$ k3: 0123456789abcdef; k6: b25286f7d3e7b3e1

\section{Conclusions and Work in Progress}

In this paper we describe the steps taken in studying the influence of various CMS configurations on the SAT solver's performance in trying to find solutions for cryptographic instances representing algebraic known-plaintext attacks on the 3 rounds small AES-64 model cipher. The static and dynamic analysis of CMS has pointed to the most computationally intensive parts, which in turn motivated variations of certain configuration parameters expected to influence the execution time of mainly these parts of the code. We also modified the source code in a way that enhances configuration changes and produces clearer results. We performed statistical runtime analysis of a plethora of results created in both default and other solver configurations which enabled us to identify solver configurations that solve the here discussed instances and thus fully recover the 64-bit key in time intervals underneath an hour (real time). ${ }^{5}$ This result is independent of the security quality of the key. By means of an Automatic Algorithm Configuration (AAC) we could even improve the previous best runtimes achieved with empirically decided configurations. Since CMS has many more parameters than varied here, it potentially offers possibilities for further configuration optimization. Therefore we intend to expand our efforts in this direction with AAC. The universality of validity of the here elaborated configurations has still to get verified. This demands the creation of many more instances and running of many more tests. Also experiments with other types (e.g. dense) of CNF-instances have to be considered. Perhaps, when appropriately tuned, CMS can solve even bigger cryptographic problems. Increasing the number of rounds means having to handle CNF-instances with many more variables and many more millions of constraints in comparison to the here handled problems. A worst case complexity would be soon formally reached. However, in the SAT solver context, worst case complexity has no explanatory or predictive power [4].

\footnotetext{
${ }^{5}$ The solution is found by one thread in this time, but not without exchange with the other threads also searching a solution in this time. In a sense one could say: CPU solution time of the winning thread.
} 


\section{References}

[1] A. Charfi. SAT-Solving in Algebraic Cryptanalysis. B. Sc. thesis, TU Darmstadt, 2014.

[2] C. Cid, S. Murphy, and M. Robshaw. Small Scale Variants of the AES. In Fast Software Encryption, pages 145-162. Springer Berlin Heidelberg, 2005.

[3] N. T. Courtois and J. Pieprzyk. Cryptanalysis of Block Ciphers with Overdefined Systems of Equations. In Advances in Cryptology - ASIACRYPT 2002, pages 267-287. Springer, 2002. An extended version of this paper is available on https://eprint.iacr.org/2002/044.

[4] Vijay Ganesh. The Impact of Community Structure on SAT Solver Performance. In Formal Foundations for Networking (Dagstuhl Seminar 15071), Dagstuhl, Germany, 2015. Schloss Dagstuhl-Leibniz-Zentrum für Informatik. https://materials.dagstuhl.de/files/15/15071/ 15071. VijayGanesh.Slides.pdf.

[5] Brendan Gregg. Flame Graphs: Visualization of Profiled Code. https://github.com/ brendangregg/FlameGraph, 2017.

[6] F. Hutter, H. H. Hoos, and K. Leyton-Brown. Sequential Model-based Optimization for General Algorithm Configuration. In Proceedings of the 5th International Conference on Learning and Intelligent Optimization, LION'05, pages 507-523. Springer Berlin Heidelberg, 2011.

[7] F. Hutter, M. Lindauer, A. Balint, S. Bayless, H. H. Hoos, and K. Leyton-Brown. The Configurable SAT Solver Challenge (CSSC). Artificial Intelligence Journal (AIJ), 243:1-25, 2017.

[8] J.-P. Indrøy. Algebraic Attack on Small Scale Variants of AES using Compressed Right Hand Sides. Master's thesis, University of Bergen, 2018.

[9] A. M. Leventi-Peetz and J.-V. Peetz. Generating and exploring S-box multivariate quadratic equation systems with SageMath. In 2017 IEEE Conference on Dependable and Secure Computing, pages 377-383, 2017.

[10] M. Lindauer, K. Eggensperger, M. Feurer, S. Falkner, A. Biedenkapp, and F. Hutter. SMAC v3: Algorithm Configuration in Python. https://github.com/automl/SMAC3, 2017.

[11] M. S. E. Mohamed, S. Bulygin, M. Zohner, A. Heuser, M. Walter, and J. Buchmann. Improved algebraic side-channel attack on AES. In 2012 IEEE International Symposium on HardwareOriented Security and Trust, pages 146-151, 2012.

[12] M. Soos, K. Nohl, and C. Castelluccia. Extending SAT Solvers to Cryptographic Problems. In Theory and Applications of Satisfiability Testing - SAT 2009, 12th International Conference, SAT 2009, Swansea, UK, June 30 - July 3, 2009. Proceedings, pages 244-257, 2009.

[13] Mate Soos. CryptoMiniSat: An Advanced SAT Solver. https://github.com/msoos/ cryptominisat, 2017. 